SESSION I. INTRODUCTION - Chair: Bambang Hidayat

"... But quite otherwise was the case of $\gamma$ Argîs when on April 24th I first viewed its spectrum in the open field of the prismatic eye-piece. Its intensely bright lines in the blue, and the gorgeous group of three bright lines in the yellow and orange, render its spectrum incomparably the most brilliant and striking in the whole heavens. ..."

Ralph D. Copeland, 1884, Copernicus 3, 193, 'Experiments in the Andes'. 


\title{
A DECADE OF WOLF-RAYET LITERATURE
}

\author{
Karel A. van der Hucht \\ SRON Space Research Utrecht \\ Sorbonnelaan 2, 3584 CA Utrecht \\ The Netherlands
}

\begin{abstract}
To set the stage for the symposium, a brief overview is given of the main thrust of the about 1250 papers on Wolf-Rayet star research published in the past decade, broken down into inventory, basic parameters and galactic distribution, atmospheres, binaries, intrinsic variability, mass loss, enrichment and evolution.
\end{abstract}

\section{Introduction}

This year we celebrate the 123rd anniversary of the discovery by Wolf and Rayet (1876) of the stars bearing their names. Before, among those stars of which astronomers started to study their brightness with the help of a prism, only one case was known, i.e. $\gamma$ Cas, of which the spectrum showed constantly bright lines. In their discovery paper Wolf and Rayet reported to l' Académie the existence of similar lines in three stars in the constellation of Cygnus, now known as WR134, WR135 and WR137, which distinguished themselves from neighbouring stars by their bright lines and yellow colours: pure yellow, orange yellow and greenish yellow, respectively. So far for the classification.

Since then about 2500 papers on Wolf-Rayet (WR) stars have been published, meaning a lot of papers under the bridge, but also many still outstanding. Those 2500 papers put together occupy less then three metres on a book shelf. Each of us makes a living by adding a few millimeters per year, some of us more than others. During the coming days we will add some three centimeters of literature to the subject, which is a fine prospect, because they will constitute a new milestone in the history of massive star research.

Figure 1 on the number of WR papers vs. time shows that since the early 60 -s the production rate has increased sharply, and that half of the total number of $2500 \mathrm{WR}$ papers has been published in past decade (van der Hucht 1990). Peaks in the distribution can readily be identified with conference years, or more often, the year after. In 1989 authors were apparently holding their breath for this symposium, which will give 1991 a kick-off with 134 papers.

In the past ten years a fair number of reviews and proceedings of conferences related to WR stars and other hot massive stars have appeared, and without claiming completeness we list here some of them:

1981, van der Hucht, Conti, Lundström \& Stenholm, 'The Sixth Catalogue of Galactic Wolf-Rayet stars, their Past and Present'; Appendix: 'A bibliography on galactic Wolf-Rayet literature, 1867-1980'. 


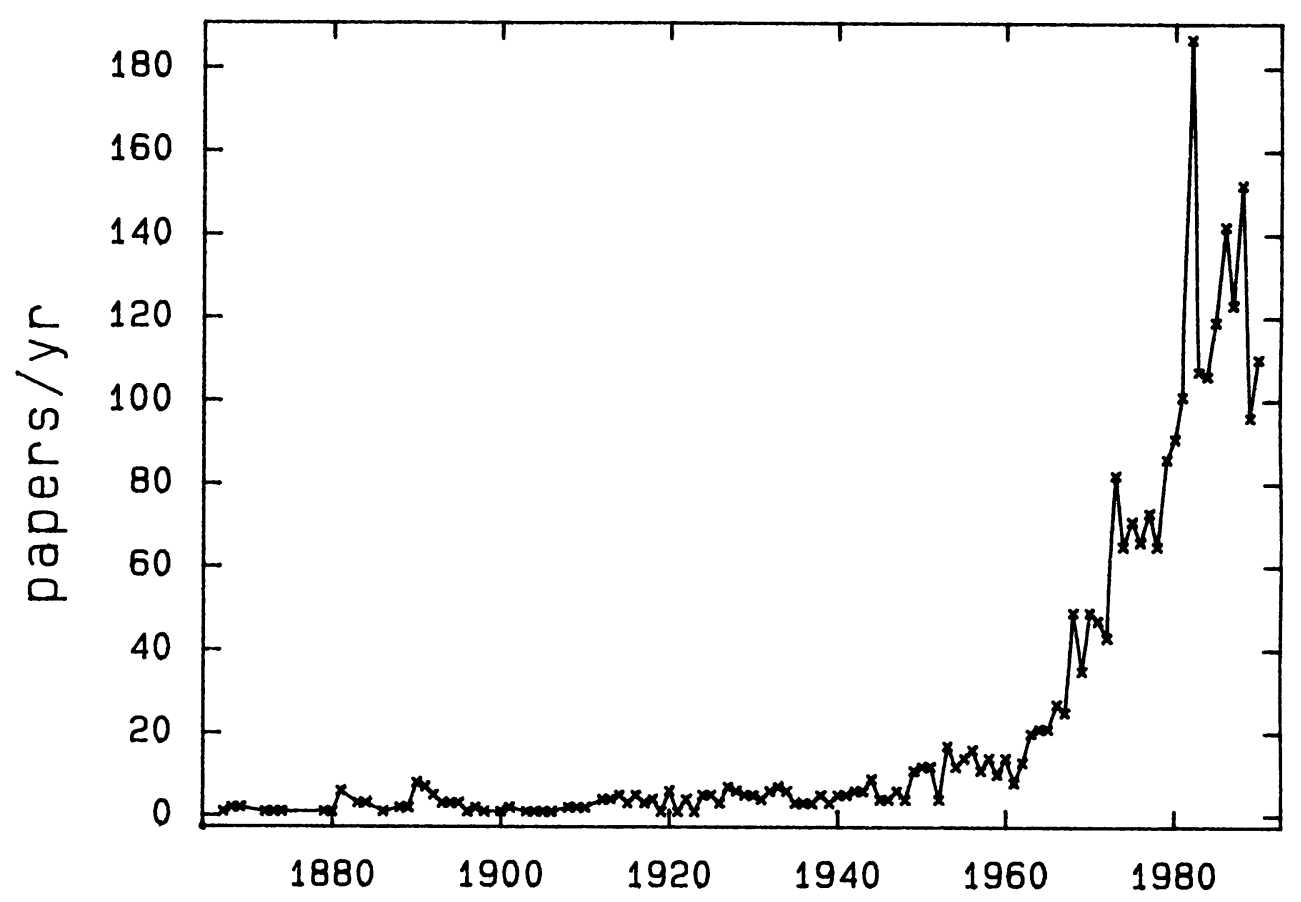

Figure 1. 123 years of Wolf-Rayet literature.

1981, D'Odorico et al. (eds.), Proc. ESO Workshop, 'The Most Massive Stars'.

1981, Chiosi \& Stalio (eds.), Proc. IAU Coll. No. 59, 'Effects of Mass Loss on Stellar Evolution'. 1982, de Loore \& Willis (eds.), Proc. IAU Symp. No. 99, 'Wolf-Rayet Stars: Observations, Physics, Evolution'.

1983, Lortet \& Pitault (eds.), Proc. Paris Workshop, 'Wolf-Rayet Stars: Progenitors of Supernovae?'

1985, Massey, 'WR stars in Nearby Galaxies'.

1986, de Loore et al. (eds.), Proc. IAU Symp. No. 116, 'Luminous Stars and Associations in Galaxies'.

1986, Chiosi \& Maeder, 'The Evolution of Massive Stars with Mass Loss'.

1987, Abbott \& Conti, 'Wolf-Rayet Stars'.

1987, Willis \& Garmany, 'Wolf-Rayet Stars'. (IUE)

1987, Lamers \& de Loore (eds.), Proc. Cornelis de Jager Workshop, 'Instabilities in Luminous Early Type Stars'.

1988, Conti \& Underhill (eds.), 'O Stars and Wolf-Rayet Stars'.

1988, Nugis \& Pustyl'nik (eds.), Proc. El'va All-Union Conference, 'Wolf-Rayet Stars and Related Objects'.

1989, Davidson et al. (eds.), Proc. IAU Coll. No. 113, 'Physics of Luminous Blue Variables'.

1990, Garmany (ed.), Proc. Boulder-Munich Workshop, 'Intrinsic Properties of Hot Luminous Stars'.

1990, Willson \& Stalio (eds.), Proc. Ames Workshop, 'Angular Momentum and Mass Loss for Hot Stars'. 
Table 1. Wolf-Rayet literature 1980 - 1989: number of papers per topic

\begin{tabular}{|l|rrrrrrrrrr|}
\hline \hline & 1980 & 1981 & 1982 & 1983 & 1984 & 1985 & 1986 & 1987 & 1988 & 1989 \\
\hline basic parameters & 14 & 9 & 16 & 14 & 13 & 11 & 13 & 12 & 9 & 6 \\
atmospheres & 17 & 11 & 61 & 21 & 16 & 28 & 20 & 13 & 26 & 21 \\
variability & 1 & 2 & 4 & 2 & 4 & 6 & 16 & 26 & 16 & 12 \\
binaries & 18 & 15 & 35 & 14 & 18 & 14 & 16 & 9 & 27 & 12 \\
mass loss & 5 & 5 & 14 & 4 & 2 & 9 & 15 & 12 & 13 & 7 \\
shells, nebulae & 10 & 17 & 17 & 14 & 14 & 11 & 12 & 8 & 16 & 9 \\
extragalactic & - & 6 & 11 & 9 & 7 & 8 & 10 & 9 & 7 & 3 \\
evolution & 15 & 26 & 24 & 24 & 29 & 29 & 34 & 29 & 26 & 25 \\
interstellar & 7 & 6 & 1 & 2 & 3 & 1 & 1 & - & 2 & - \\
review or book & 4 & 4 & 4 & 3 & - & 2 & 4 & 5 & 10 & 1 \\
\hline total & 91 & 101 & 187 & 107 & 106 & 119 & 142 & 123 & 152 & 96 \\
\hline \hline
\end{tabular}

The distribution of papers over the various topics as listed in Table 1 has generally been stable, with always the largest fraction devoted to evolutionary studies. But there are certain features. E.g., 1987 shows a sudden peak in variability studies (a topic of increasing interest in the 80-s) at the expense of studies of (alleged) binaries, though the next year binaries are up again. Extragalactic studies seem to be reaching, for the time being, technical limits at the end of the 80 -s.

To set the stage for the symposium, we briefly overview the status quo of the various topics.

\section{Inventory}

In the Galaxy the WR census by van der Hucht et al. (1988) has been complemented with the discovery of one new WR star, listed by The (1964) as planetary nebula Th3-28, but classified as a new WN2.5-3 star without a nebula by Acker \& Stenholm (1990); with the discovery by Cohen et al. (1991 and these proceedings) of IRAS 17380-3031 as a new WC 9 star; and with the discovery of 13 new WR stars (11 WN and 2 WC) by Shara et al. (these proceedings). This brings the number of known galactic WR stars to 172 , among which there are $91 \mathrm{WN}, 6 \mathrm{WN} / \mathrm{WC}$ (Conti \& Massey 1989), $73 \mathrm{WC}$ and 2 WO (Barlow \& Hummer 1982) stars.

For the LMC, Lortet (these proceedings) refers to 27 new WR stars since the census by Breysacher (1981). Excluding the 12 Of/WN stars she lists, this brings the number of LMC WR stars up to 115. Of particular importance among the new LMC WR stars is one WC9-type (Heydari-Malayeri et al. 1990 and these proceedings), a subtype which was hitherto unknown in the LMC.

In the SMC no new WR stars have been found since the census by Azzopardi \& Breysacher (1979), keeping the number at 8, among which there are $7 \mathrm{WN}$ and 1 WO stars.

A new topic of the past decade form the numerous discoveries of extragalactic WR stars, in Table 2 broken down into the Local Group and beyond. Only in Local Group galaxies WR stars have been studied individually, as reviewed, e.g., by Massey (1985). In more remote galaxies, primarily irregular dwarf galaxies and blue compact emission line galaxies, 
Table 2. Wolf-Rayet stars in galaxies

\begin{tabular}{|c|c|c|c|c|c|}
\hline $\begin{array}{l}\text { Local Group } \\
\text { galaxies }\end{array}$ & $\begin{array}{r}\mathrm{d} \\
(\mathrm{kpc})\end{array}$ & type & $\mathrm{N}(\mathrm{WR})$ & $\begin{array}{c}\text { subtype } \\
\text { (WC/WN) }\end{array}$ & References \\
\hline Milky Way & & $\mathrm{Sb} / \mathrm{c}$ & 172 & 0.77 & $14,31,62$ \\
\hline LMC & 52 & Irr I & 115 & 0.26 & $6,29,43,53$ \\
\hline SMC & 63 & Irr I & 8 & 0.14 & 5 \\
\hline NGC 6822 & 470 & Irr & 2 & WN & $2,7,16,49,51,58,64,67$ \\
\hline M31 = NGC 224 & 670 & $\mathbf{S b}$ & 46 & 2.8 & $3,16,32,47,49,51,52,61,64$ \\
\hline - NGC 206 & & & 11 & & 47,52 \\
\hline M33 = NGC 598 & 730 & Scd & 96 & 0.7 & $7,8,16,17,28,42,45,48,49,59,64,66,68$ \\
\hline - NGC 588 & & & & & 15,58 \\
\hline - NGC 592 & & & & & 15,58 \\
\hline - NGC 595 & & & 9 & & $15,25,58$ \\
\hline - NGC 604 & & & 50 & WN7 & $13,15,21,22,25,30,54,57$ \\
\hline - IC 132 & & & & WNE & 9,23 \\
\hline IC 1613 & 740 & Irr I & 1 & WN & $2,7,16,18,24,42,49,64$ \\
\hline $\begin{array}{l}\text { beyond the } \\
\text { Local Group }\end{array}$ & $\begin{array}{r}d \\
(\mathrm{Mpc})\end{array}$ & type & $\mathrm{N}(\mathrm{WR})$ & $\begin{array}{c}\text { subtype } \\
\text { (WC/WN) }\end{array}$ & References \\
\hline C1148-203 & & BC & 10000 & WNE & 11,12 \\
\hline ESO 148-IG02 & 200 & pec & many & WNL & 35 \\
\hline Haro 2 & & BC & & & 44 \\
\hline $\begin{array}{l}\mathrm{He} 2-10=\mathrm{ESO} \\
\mathrm{IC}\end{array}$ & 1.5 & Irr II & 3300 & 2.2 & $1,22,33,34,38,54,69$ \\
\hline $\begin{array}{l}\text { IC } 4662 \\
\text { IRAS 01003-2238 }\end{array}$ & 470 & $\begin{array}{c}\text { Irr } \\
\text { FIR }\end{array}$ & 100000 & WNL & $\begin{array}{c}59 \\
4\end{array}$ \\
\hline ISZ 59 & 25 & BC & & & 38 \\
\hline M83 = NGC 5236 & 3.2 & Sbc & 180 & WN,WC & 59,60 \\
\hline M101 = NGC 5457 & 3.8 & Scd & & & \\
\hline - NGC 5447 & & & & & 22,58 \\
\hline - NGC 5455 & & & & & 22,58 \\
\hline — NGC 5461 & & & & WN6-7 & $22,55,58$ \\
\hline - NGC 5471 & & & & & $41,44,58,63$ \\
\hline - Ho 40 & & & & WN7 & 22 \\
\hline - Searle 12 & & & & & 22 \\
\hline Mkn 33 & 24 & BC & & & 38 \\
\hline Mkn 59 & & $\mathrm{BC}$ & & & 44 \\
\hline Mkn 309 & 160 & $\mathrm{BC}$ & 50000 & 1.5 & 38,54 \\
\hline Mkn 724 & 18 & BC & 120 & WN4-5 & 40 \\
\hline Mkn 750 & 11 & $\mathrm{BC}$ & & & 38 \\
\hline Mkn 1236 & 26 & $\mathrm{BC}$ & 900 & & 40 \\
\hline NGC 55 & 2.3 & Sd-Irr & & & 59 \\
\hline NGC 300 & 1.4 & $\mathrm{Sc}$ & & & $19,22,47,59$ \\
\hline - \#2 & & & & WC5 & 22 \\
\hline - \#5 & & & & & 22 \\
\hline -\#7 & & & & WN & 22 \\
\hline -\#13 & & & & & 22 \\
\hline -\#24 & & & & WC & 19 \\
\hline -\#53c & & & & WN & 19 \\
\hline -\#77 & & & & WN & 19 \\
\hline -\#137D & & & & WC & 19 \\
\hline NGC $1741=$ Mkn 1089 & 58 & pec & 19500 & WN & 40 \\
\hline NGC $3049=$ Mkn 710 & 20 & SBbp & 15700 & WN & 40,44 \\
\hline NGC $3125=$ Tol 3 & 17 & $\mathrm{Sa}$ & $300-10000$ & WN & $12,38,39,44,54,58$ \\
\hline
\end{tabular}


Table 2 (cont'd)

\begin{tabular}{|c|c|c|c|c|c|}
\hline $\begin{array}{l}\text { beyond the } \\
\text { Local Group }\end{array}$ & $\begin{array}{r}d \\
(\mathrm{Mpc})\end{array}$ & type & N(WR) & $\begin{array}{c}\text { subtype } \\
\text { (WC/WN) }\end{array}$ & References \\
\hline NGC 4038 & & Sd-Irr & & & 59 \\
\hline NGC 4214 & & Irr & & & 44 \\
\hline NGC 4216 & & Sb & & & 59 \\
\hline NGC $4385=$ Mi $499=$ Mkn 52 & 50 & SBbc & $1300 / 4200$ & WN/WC8 & 11,27 \\
\hline NGC 4670 & & Irr & & & 44 \\
\hline NGC 4861 & 9 & Irr & & & 20 \\
\hline NGC 5068 & & Sc & & & 59 \\
\hline NGC $5128=$ Cen $A$ & 4.4 & SO & & & $50,58,59$ \\
\hline NGC 5253 & & E-SO & 10000 & WNE & $11,12,59$ \\
\hline NGC 5398: Tol 89 & 20 & Sd & 180 & WN7-8 & 26,38 \\
\hline NGC $5430=$ Mkn 799 & 60 & SBb & 30000 & & 36,37 \\
\hline NGC 6764 & 480 & Sey & 3000 & 2.0 & 38,54 \\
\hline Pox 4 & 51 & BC & & & 38,39 \\
\hline Pox 120 & 89 & BC & & & 38 \\
\hline Pox 139 & 30 & $\mathrm{BC}$ & & & 38 \\
\hline Pox 186 & 15 & $\mathrm{BC}$ & & & 38 \\
\hline Tol 2 & 15 & BC & & & 38 \\
\hline Tol 9 & 52 & BC & & WN,WC & 40 \\
\hline Tol $35=\mathrm{T} 1324-276$ & 24 & $\mathrm{BC}$ & & WNE & 11,38 \\
\hline IZw18 & 10 & BC & & & 41,58 \\
\hline IIZw33 & 39 & BC & & & 38 \\
\hline IIZw40 & & BC & & & 39 \\
\hline IIZw62 & 56 & BC & & & 38 \\
\hline IIZw 187 & 169 & BC & & & 38 \\
\hline IIIZw107S & 79 & BC & & & 38 \\
\hline VIIZw19 & 69 & BC & & & 38 \\
\hline VIIZw156NE $=$ Mkn 8 & 49 & BC & & & 38 \\
\hline
\end{tabular}

References: 1. Allen et al. 1976; 2. Armandroff \& Massey 1985; 3. Armandroff et al. 1986; 4. Armus et al. 1988; 5. Azzopardi \& Breysacher 1979; 6. Azzopardi \& Breysacher 1985; 7. Azzopardi et al. 1988; 8. Bohannan et al. 1985; 9. Boksenberg et al. 1977; 10. Breysacher 1981; 11. Campbell \& Smith 1986; 12. Campbell et al. 1986; 13. Clayton 1988; 14. Cohen et al. 1991 and these proceedings; 15. Conti \& Massey 1981; 16. Conti 1988; 17. Corso 1975; 18. Davidson \& Kinman 1982; 19. Deharveng et al. 1988; 20. Dinerstein \& Shields 1986; 21. D'Odorico \& Rosa 1981; 22. D'Odorico et al. 1983; 23. D'Odorico \& Benvenuti 1983; 24. D'Odorico \& Rosa 1982; 25. Drissen et al. 1989a; 26. Durret et al. 1985; 27. Durret \& Tarrab 1988; 28. Freedman 1985; 29. Heydari-Malayeri et al. 1990; 30. Hippelein \& Fried 1984; 31. van der Hucht et al. 1988; 32. Hutchings et al. 1987; 33. Hutsemekers \& Surdej 1984; 34. Johansson 1987; 35. Johansson \& Bergvall 1988; 36. Keel 1982; 37. Keel 1987; 38. Kunth \& Joubert 1985; 39. Kunth \& Sargent 1981; 40. Kunth \& Schild 1986; 41. Lequeux et al. 1981; 42. Lequeux et al. 1987; 43. Lortet, these proceedings; 44. Mas-Hesse \& Kunth, these proceedings; 45. Massey \& Conti 1983; 46. Massey $1985 ; 47$. Massey et al. 1986; 48. Massey et al. 1987b; 49. Massey et al. 1987a; 50. Möllenhoff 1981; 51. Moffat \& Shara 1983; 52. Moffat \& Shara 1987; 53. Morgan \& Good 1990; 54. Osterbrock \& Cohen 1982; 55 . Rayo et al. 1982; 56. Rosa \& D'Odorico 1982; 57. Rosa \& Solf 1984; 58. Rosa et al. 1984; 59. Rosa \& D'Odorico 1986; 60. Rosa \& Richter 1988; 61. Shara \& Moffat 1986; 62. Shara et al., these proceedings; 63. Skillman 1985; 64. Smith 1988; 65. Terlevich \& Melnick 1985; 66. Wampler 1982; 67. Westerlund et al. $1983 ; 68$. Wray \& Corso 1972. 69. Zinnecker, 1987. 
only integral WR features are found, though sometimes seen grouped into individual (giant) $H I I$ regions. The inferred numbers of WR stars are based on, given the distance of a galaxy, the amount of energy in its $\mathrm{HeII} \lambda 4686$ emission line compared to that of a single (galactic) WR star. Properties of extragalactic WR features will be discussed in section 9 .

\section{Basic parameters and galactic distribution}

Intrinsic parameters of Wolf-Rayet stars, like intrinsic colours, colour excesses and absolute visual magnitudes, have been derived and discussed by Hidayat et al. $(1982 ; 1984)$, Lundström \& Stenholm (1984a), Massey (1984), van der Hucht et al. (1988), Torres-Dodgen \& Massey (1988) and Vacca \& Torres-Dodgen (1990). New methods, based on emission line strengths and ratios, have been developed by Conti \& Massey (1989), Conti \& Morris (1990) and Smith et al. (1990) to determine absolute visual magnitudes of WN stars, interstellar reddening of WN stars and distances of WC stars, respectively.

Hidayat et al. $(1982,1984)$ and van der Hucht et al. (1988) used available filter photometry of Smith (1968a) and Lundström and Stenholm (1979, 1984a), which has some emission line contamination in particular for WC stars. They derived colour excesses, intrinsic colours and absolute visual magnitudes for the Galaxy only, adopting distances and colour excesses found in studies of WR stars in galactic open clusters and associations by Lundström \& Stenholm (1984a) as the basis of their calibration. In spite of the limited number of WR stars in galactic clusters and associations (42, distributed over $18 \mathrm{WR}$ subtypes), van der Hucht et al. (1988) warn against a priori mixing of galactic and LMC basic parameters. Their fear is confirmed by Hamann (these proceedings), who finds from quantitative spectroscopy that LMC WN stars have on the average lower luminosities than their galactic counterparts.

Massey (1984) and Torres-Dodgen \& Massey (1988) were able to derive synthetic line-free photometry from spectrophotometry. In addition, Vacca \& Torres-Dodgen (1990) derived colour excesses by nulling the $2200 \AA$ feature in low resolution $I U E$ spectra of galactic and LMC WR stars. The latter averaged the parameters found per subclass in the Galaxy (based on nulling for the colour excesses and on cluster and association distances for the absolute visual magnitudes) and in the LMC (based on nulling and on the LMC distance).

Studies of the galactic distribution of WR stars as a diagnostic for the determination of their initial masses and (subtype) evolution have been attempted by Maeder et al. (1980), Hidayat et al. (1982), Meylan \& Maeder (1983) and Hidayat et al. (1984) on the basis of the galactic data of van der Hucht et al. (1981); by Conti et al. (1983) based on the LMC data of Prévot-Burnichon (1981); by van der Hucht et al. (1988) using galactic data only; and by Conti \& Vacca (1990) mixing galactic and LMC data. In all those studies the overall galactic WR distributions found agree remarkably well among each other, in spite of the different data sets and methods used. Notably the concentration of WR stars in galactic spiral arms and their absence toward the galactic anti-center (Orion spur) as known since the work by Roberts (1962), and the concentration of late-WC stars toward the galactic center as known since the work by Smith (1968b), are confirmed by the recent studies.

Conti \& Vacca (1990), using WR absolute visual magnitudes determined by Vacca \& Torres (1990) by averaging galactic data (from cluster and association distances) and LMC data, re-calculated the distances of the galactic WR stars first used as members of open clusters and associations, which constitute about $50 \%$ of the number of WR stars within $2.5 \mathrm{kpc}$ from the Sun. This inconsistency causes the main discrepancy between their WR 
galactic distribution and the one found by van der Hucht et al. (1988), the latter showing better agreement in subtype ratios vs. galactocentric distance with evolutionary calculations of Maeder (1990b and these proceedings). Comparison between the two observational studies also indicates that distances of WR field stars are presently determinable with an accuracy of only about $50 \%$.

\section{Atmospheres}

Spectrophotometric atlases of WR stars have become available from the ultraviolet to the infrared. Ultraviolet (IUE; no single observatory ever had such a profound effect on hot star research) low and high resolution atlases have been published by Nussbaumer et al. (1982) and Willis et al. (1986), respectively, atlases of low resolution IUE spectra combined with optical spectra have been given by Smith and Willis (1983) for LMC WR stars, and by Garmany et al. (1984) for galactic WR stars. Optical spectral atlases have been presented by Smith \& Kuhi (1981), by Sivertsen (1981), by Lundström \& Stenholm (1984b,c), by Jeffers \& Weller (1985), by Torres \& Conti (1984) for WC9 stars, by Torres \& Massey (1987) for WC stars, and by Lundström \& Stenholm (1989). Near-infrared spectral atlases have been published by Vreux et al. (1983), Vreux et al. (1989), Conti et al. (1990) and Vreux et al. (1990).

Infrared photometric fluxes originating in WR stellar winds or, for the late-WC types, in their circumstellar dust shells are discussed in sections 7 and 8 , respectively. Free-free radio and normal $\mathrm{X}$-ray fluxes are related to stellar winds in section 7 , while non-thermal radio and strong $\mathrm{X}$-ray fluxes are related to colliding winds in binaries in section 5 .

The development of model atmospheres for WR stars has taken great strides in recent years, as demonstrated in reviews by Hamann et al. (1990), Hillier (1990) and Schmutz (1990), in turn reviewed by Kudritzki \& Hummer (1990). Multilevel non-LTE codes have been developed for hot stratified expanding pure helium model atmospheres by Hamann \& Schmutz (1987), Hamann et al. (1988), Schmutz et al. (1989) and Hillier (1987a,b, 1988, 1989), the latter two papers also including nitrogen and carbon. Those models, using the Sobolev approximation and treating line and continuum formation simultaneously, are able to calculate continuum energy distributions, line-strengths and line-profiles for WN and WC stars, which match observations to a satisfactory degree of accuracy. They prove that the WN and WC sequences arise from different surface abundances of He and CNO, thus confirming the suggestion of Gamow (1943) that WN and WC stars display at their surfaces products of different phases of thermo-nuclear processing.

Typical number abundance ratios derived from those detailed model atmospheres in comparison with observations are $\mathrm{N} / \mathrm{He} \approx 4 \times 10^{-3}$ and $C / N \approx 0.07$ for the WN5 star WR6 (Hillier 1988) and $C / H e>0.1$ for the WC5 star WR111 (Hillier 1989). Smith \& Hummer (1988) and Smith \& Maeder (1990) find a continuous increase in the $(C+O) / H e$ number ratio for the sequence WCL $\rightarrow$ WCE $\rightarrow$ WO, ranging from 0.03 to $>1$.

The effective temperatures found by comparison between pure helium model atmospheres and observations range from 30 to $35 \mathrm{kK}$ for WNL and WC stars, and from 35 to $90 \mathrm{kK}$ for WNE stars (Schmutz et al. 1989), while higher temperatures for WC stars are found when carbon is introduced (Hillier 1989). Such temperatures have been confirmed independently for WR stars with ring nebulae (Rosa \& Mathis 1990). Corresponding bolometric corrections range from $-3 \mathrm{mag}$ for model atmospheres with some hydrogen content to $-5 \mathrm{mag}$ for model atmospheres without hydrogen. This corresponds reasonably well with average B.C. 
values of $-4.2 \mathrm{mag}$ derived by van der Hucht et al. (1988) and of -4.5 mag derived by Smith \& Maeder (1989) from studies of WR binaries in open clusters and associations, inferring their luminosities from their observed masses and adopted theoretical $M$ - $L$ relations for evolved massive stars, e.g., by Maeder \& Meynet (1987). These results lend additional confidence to the reliability of the model atmospheres and the interior models used, to the WR cluster/association memberships adopted, as well as to the reality that almost all WR stars are hydrogen deficient.

At variance with the results mentioned above, which demonstrate that WR stars are evolved massive stars with larger-than-cosmic abundances of helium and nitrogen in the atmospheres of WN stars and of helium, carbon and oxygen in the atmospheres of WC stars, are the conclusions of Underhill (1990 and references therein). She favours cosmic abundances and a pre-MS evolutionary status for WR stars, and argues that the observed differences in line strength of nitrogen and carbon lines in WN and WC stars are caused by different wind densities and electron temperatures and not by differences in surface abundances. Her one-representative-point models have been refuted, e.g., by Nugis (these proceedings) who by applying her models found discrepancies of an order of magnitude between calculated and observed $C I I I$ and $C I V$ line ratios. Arguments proving the evolved evolutionary status of WR stars, like: (i) the lack of hydrogen in most WR stars as found in their Pickering decrement (Smith 1973); (ii) the WR atmospheric abundance determinations mentioned above and the high nitrogen content of WR ring nebulae (e.g., Rosa \& Mathis 1990); (iii) the abundance predictions by interior models of, e.g, Maeder \& Meynet (1987) and Maeder (1990b), which also match the observed WR/O star number ratios in the Galaxy (van der Hucht et al. 1988); and (iv) the fact that some WR binaries have evolved companions; have been reiterated by Lamers et al. (1990). Even more compelling arguments in favour of the evolved evolutionary status of WR stars are ( $v$ ) the continuous carbon dust formation around WC stars only (Williams et al. 1987); and (vi) a fractional abundance $n_{C} \approx 0.06$ derived from the X-ray spectrum of the WC7 binary WR140 (Williams et al. 1990a), further discussed in section 5 .

\section{Binaries}

After the numerous studies of WR binaries in the early 80-s by Massey (1981) and coworkers, the main breakthrough has been the determination of orbital inclinations by Moffat and co-workers from linear polarization modulation measurements, as summarized by St.Louis et al. (1988), Moffat (1988) and Schulte-Ladbeck \& van der Hucht (1989). Resulting masses listed by those authors range from 6 to $40 M_{\odot}$ for WN stars and from 5 to $20 M_{\odot}$ for WC stars in binaries. Other new studies of established binaries (i.e., those with radial velocity solutions) have been summarized, e.g., by Smith \& Maeder (1989) for the Galaxy and by Moffat et al. (1990) for the LMC and SMC.

WR stars which may be accompanied by a compact companion left after a supernova explosion of the primary, $c f$. the evolutionary scheme of van den Heuvel (1976), are listed by Hidayat et al. (1984). Their number has dwindled somewhat since, where the interest has drifted from compact companions to intrinsic variability (cf. Vreux 1985). Among the dozen or so left, the two most likely candidates are still WR6 (WN5 + neutron star?, $\mathrm{P}=3.76 d)$ (Firmani et al. 1980; Drissen et al. 1989b; van der Hucht et al. 1990) and WR148 (WN7 + black hole?, $\mathrm{P}=4.32$ d) (Drissen et al. 1986).

The binary frequency in the solar neighborhood was found to be $37 \%$ (van der Hucht et 
al. 1988), and this number is still increasing. A fascinating but time consuming new way of detecting binaries has been discovered by Williams et al. $(1985,1990 \mathrm{a}, \mathrm{b}$, these proceedings) in the case of long period $(\mathrm{P} \approx 10 \mathrm{yr})$ eccentric $\mathrm{WC}$ binaries with periodic dust formation (section 8 ). Because of their relatively large component separations, these binaries can also be discovered as strong X-ray and non-thermal radio sources, which emissions are believed to be associated with their colliding winds (Williams et al. 1990a). From their $\mathrm{X}$-ray luminosity and non-thermal radio characteristics, also possible WN binaries with large separations may be discovered, for which WR25 (WN7+a) (Pollock 1987 and these proceedings) and WR147 (WN7) (Caillaut et al. 1985; Moran et al. 1989) are likely candidates.

Studies of colliding winds have also been performed for the WR binary V444 Cyg (WR139, WN5+06, $\mathrm{P}=4.21 d$ ) by Shore \& Brown (1988), explaining the variability of its UV P-Cygni profiles in terms of shock dominated wind-wind interactions, and by Luo et al. (1990) and Usov (1990), addressing its X-ray luminosity modulation.

New short-period binaries $(\mathrm{P}<1 d)$ may be discovered from high time-resolution photometric observations as demonstrated, e.g., by van Genderen et al. (1990 and these proceedings), although also intrinsic variability may play a role here (section 6 ).

\section{Intrinsic variability}

Interior models of WR stars indicate that the fundamental radial mode could be excited due to nuclear-burning instabilities (Maeder 1985; Cox \& Cahn 1988) with periods of about 1 to $2 \mathrm{hr}$. Non-radial modes, which have longer periods, have found to be generally stable. Vreux $(1985,1987)$ attacked the credibility of published periods of emission-line profile variations of alleged $W R+c$ systems, and suggested the observed variability to be due to single-star non-radial pulsations. In order to find radial or non-radial pulsations in WR stars, observations have to be of a quality which allows searches for periods of less than one day and for multiple periodicities.

From intensive photometry encouraging new results have become available. E.g., Taylor et al. (1988) and Taylor (1990) find a $\mathrm{P}=1.26 d$ in $\gamma^{2}$ Vel (WC8+09I); Gosset \& Vreux (1990) find $\mathrm{P}_{A}=6.25 d$ and $\mathrm{P}_{B}=2.5 d$ in WR40 (WN8); Gosset et al. (1990) find $\mathrm{P}_{A}=9.09 d, \mathrm{P}_{B}=15.15 d$, and $\mathrm{P}_{C}=5.46 d$ in WR16 (WN8); and van Genderen et al. (1990 and these proceedings) find tentatively $\mathrm{P}=0.28 d$ for WR46 (WN3p), $\mathrm{P}=1.06 d$ for WR50 (WC6+a), $\mathrm{P}=2.44 d$ for WR55 (WN7), and P = $1.94 d$ for WR123 (WN8); but for each of these stars duplicity as the cause of the observed variability can not be excluded. Also Balona et al. (1989) present a number of cases.

From intensive spectroscopic observations of line-profile variations, e.g., Vreux et al. (1985) find $\mathrm{P}_{A}=0.45 d$ and $\mathrm{P}_{B}=0.31 d$ in WR136 (WN6+c?); for the same object St.Louis et al. (1989) find $\mathrm{P} \approx 1 d$ from $I U E$ high resolution monitoring; and Willis et al. (1989) find $\mathrm{P} \approx 1 d$ for WR6 (WC5+c?), also with $I U E$.

Variability indicative of blob ejection in the winds of WR stars was suggested first from photometry by, e.g., van Genderen et al. (1987) and has subsequently been confirmed in high-time- and high-spectral resolution spectroscopy by Moffat et al. (1988), McCandliss (1988) and Robert \& Moffat (1990). Linear polarization variability, possibly also related to blob ejection, has been reported by Moffat (1988, and references therein) and SchulteLadbeck \& van der Hucht (1989), and appears to be largest in the late-WR subclasses. 


\section{Mass Loss}

Wind characteristics and mass loss rates of WR stars have been elaborated on by Willis (1981), updated by Willis \& Garmany (1987 and references therein). Mass loss rates can be determined for many WR stars from IR (Barlow et al. 1981) and radio (Abbott et al. 1986) free-free measurements for the electron densities, from UV P-Cygni profiles for the wind terminal velocities (Willis \& Garmany 1987), and from their distances, applying the theory of spherically symmetric mass loss by Wright \& Barlow (1975).

In the past years such determinations have been affected by the following realizations: (i) a high carbon abundance and a low ionization leads to a higher mass loss rate, especially for late-type WC stars (van der Hucht et al. 1986); (ii) an outward decreasing ionization degree leads to a higher mass loss rate (Schmutz \& Hamann 1986; Hillier 1987a); (iii) improved distances (e.g., by van der Hucht et al. 1988) are of importance because $\dot{M} \propto d^{3 / 2}$; and (iv) $C I V \lambda 1550$ edge velocities yield overestimates of terminal velocities (Williams \& Eenens 1989; Prinja et al. 1990), leading to lower mass loss rates. Taking those effects into account, Prinja et al. (1990) derive mass loss rates for WR stars in the range $\dot{M}=[2-$ 10] $\times 10^{-5} M_{\odot} y r^{-1}$.

A new method to derive the electron density in the winds of WR binaries from polarization modulation measurements has been developed by St.-Louis et al. (1988), confirming the electron densities derived from radio free-free measurements. Since the same observations yield also orbital inclinations (section 5), together they allow a consistent observational determination of a relation between mass and mass loss. An overall dependence $\dot{M} \propto M^{1-2}$ seems compatible with most of the data (St.-Louis et al. 1988).

An independent method to find mass loss rates of WR binaries from observed period changes has been developed and applied to V444 Cyg (WN5+O6) by Khaliullin (1974) and has been improved by Khaliullin et al. (1984). The latter determined that $\dot{M}=1.0 \times 10^{-5} M_{\odot} y r^{-1}$, whereas Prinja et al. (1990) found for the same star $\dot{M}=2.4 \times 10^{-5} M_{\odot} y r^{-1}$ by applying the Wright \& Barlow method. From her own data set of V444 Cyg, Underhill et al. (1990) find a $60 \%$ smaller period change and a $50 \%$ smaller mass loss rate than found by Khaliullin et al. (1984). Since this method has been applied up to now for just one WR binary, its results can not be generalized to be representative for all WR stars.

WR stars have the largest constant mass loss rates of all stellar classes. A problem not solved in the 80-s is that of the WR wind momentum, conveniently expressed in the ratio $\eta=\left(\dot{M} v_{\infty}\right) /\left(L_{\star} / c\right)$. Although the problem has come down from $\eta=5-50$ (Barlow et al. 1981) to $\eta=1-30$ (Willis, these proceedings), it is not clear which mechanism, apart from radiation pressure, drives the WR stellar winds. A possible way out has been offered by Poe et al. (1989), involving effects of rotation, differences in equatorial and polar outflows and open magnetic fields, but needs further development and observational evidence. Another solution, involving a pure radiation driven wind, is suggested by Bandiera \& Turolla (1990), who require a $\dot{M} \propto M^{2.3}$ dependence.

\section{Enrichment}

Enrichment by WR stars of the interstellar medium in energy and mass has been discussed by Abbott (1982) and was up-dated by van der Hucht et al. (1986). During its lifetime a WR star emits an amount of energy comparable to that released during a supernova event. 
Although, within $3 \mathrm{kpc}$ from the Sun, they comprise less than $5 \%$ of the total number of early-type stars having stellar winds (B- and A-type supergiants, O-type and WR stars), WR stars provide more than half of the mass and wind energy input into the interstellar medium.

Infrared photometric studies of WR stars since Allen et al. (1972) have demonstrated that many late-type WC stars have dust characteristics on top of their free-free energy distributions. Energy distributions combining groundbased IR photometry and IRAS-LRS spectra of late-type WC stars are presented by van der Hucht et al. (1985). Williams et al. (1987) show that $85 \%$ of the WC9 stars and $50 \%$ of the WC8 stars have heated $\left(T_{d} \approx 1300 \mathrm{~K}\right)$ circumstellar amorphous carbon dust (soot), which is being formed continuously at typical radii of $\sim 350 R_{\star}$ for WC9 stars and $\sim 800 R_{\star}$ for WC8 stars, i.e., within their stellar winds. They argue that a critical wind density $\rho \approx 8 \times 10^{-18} \mathrm{gcm}^{-3}$, corresponding to $n \approx 6 \times 10^{5} \mathrm{~cm}^{-3}$, needs to be exceeded for dust to form at distances from a central WC star where its radiation field is sufficiently diluted to allow dust formation. This explains why stars earlier than WC8 generally do not show dust formation: their wind densities at radii where their radiation fields would generally allow dust formation are below the critical wind density given above. Exceptions are provided by some long period binaries with eccentric orbits, like the WC7 binaries WR137 (Williams et al. 1985) and WR140 (Williams et al. 1990a), the possible WC8 binary WR48a (Williams et al. and these proceedings) and the possible WC4 binary WR19 (Williams et al. 1990b and these proceedings). In those cases local density increments during periastron passage are believed to give rise to periodic c.q. episodic dust formation. In WC9 stars with very pronounced dust characteristics, the $7.7 \mu \mathrm{m} \mathrm{PAH}$ emission feature, attributed to $C C$ stretching modes in carbonaceous materials, has been detected (Cohen et al. 1989).

The formation of amorphous carbon in the winds of late-type WC stars is a clear indication of the larger-than-cosmic carbon abundance of WC stars, and can directly be compared to cases of carbon dust formation in other evolved objects like novae (Sedlmayr \& Gass, these proceedings).

Some $10 \%$ of the known galactic and LMC WR stars are surrounded by shell nebulae, more often called ring nebulae, (semi-)spherical HII regions with radii of the order of parsecs, as a manifestation of past mass release of their central stars, be it steady wind or episodic events. Reviews have been given by Chu et al. (1983), Rosado (1986), Dufour (1989) and Smith (1990). WR ring nebulae closely resemble the symmetric $H I I$ regions seen around some Luminous Blue Variables (LBV), e.g., AG Car (Dufour 1989), and those seen around some Of stars (Lozinskaya 1982; Rosado 1986), in one case (NGC 6164/5) being bipolar (Leitherer \& Chavarria 1987; Dufour et al. 1988). It is well possible that at least the ejecta-type WR ring nebulae were created during a pre-WR (e.g., LBV) phase.

From their morphology, WR ring nebulae have been classified $W$-type (stellar wind blown bubble), $E$-type (stellar ejecta), $R_{s}$-type (shell structured $H I I$ region), and $R_{a}$-type (amorphous $H I I$ region). Most $W$-type nebulae are associated with WNE stars; most $R_{a^{-}}$ type nebulae surround WNL stars; most $E$-type nebulae surround WN8 stars; and most $R_{s}$ type nebulae surround WC stars (Chu et al. 1983).

Matter in WR ring nebulae can be a combination of (pre-WR) stellar wind c.q. ejecta and swept-up interstellar matter. Since Kwitter $(1981,1984)$, as confirmed again by Rosa \& Mathis (1990), it is known that WR ring nebulae are chemically enhanced in helium and 
nitrogen, revealing the larger-than-cosmic abundances of the (pre-)WR wind and ejecta. Observed ionization ratios in the nebulae yield effective temperatures for the central stars (Rosa \& Mathis 1990) which are in good agreement with those found by comparing WR spectra with model atmospheres (Schmutz et al. 1989). IRAS imagery shows that the dust in WR ring nebulae has a bi-modal composition, i.e., consisting mainly of large cool particles (Van Buren \& McCray 1988), but mixed with less cool small particles (Cassinelli et al., these proceedings). Recent ring nebula studies concentrate on their dynamics and energetics, e.g., Chu (1988) and Smith et al. (1988).

Because of their production of heavy elements, WR stars may to a certain degree be reponsible for anomalous $\mathrm{C}, \mathrm{Ne}$ and $\mathrm{Mg}$ isotope ratios found in cosmic rays and certain meteorites (Maeder 1983). The importance of a possible WR contribution to the amount of ${ }^{26} \mathrm{Al}$ near the galactic center, derived from the intensity of the observed $1.81 \mathrm{MeV} \gamma$-ray emission observed in that direction, is still a matter of debate, $c f$. Blake \& Dearborn (1989) and Signore \& Dupraz (1990).

\section{Evolution}

Since the review of Chiosi \& Maeder (1986) on the evolution of single massive stars and the productions of WR stars, arguing for the evolutionary sequences

$\mathrm{O} \rightarrow \mathrm{Of} \rightarrow \mathrm{BSG}$ and $\mathrm{LBV} \rightarrow \mathrm{WR} \rightarrow \mathrm{SN}$ for $M_{i}>60 M_{\odot}$ and

$\mathrm{O} \rightarrow \mathrm{BSG} \rightarrow \mathrm{RSG} \rightarrow \mathrm{WR} \rightarrow \mathrm{SN} \quad$ for $25 M_{\odot}<M_{i}<60 M_{\odot}$

(the supernovae most likely being of Type Ib, cf. Ensman \& Woosley 1988), and since the comparative study of de Loore (1988), new major evolutionary studies have appeared, most recently those of Langer $(1989 \mathrm{a}, \mathrm{b}, 1990)$ and Maeder $(1990 \mathrm{a}, \mathrm{b})$. The authors differ in that the former applies mass loss and semi-convection (the effect of molecular weight gradients on convection) as mixing process to bring nuclear burning products to the surface, while the latter relies on mass loss and convective overshooting (the Schwarzschild criterion and an increase of the convective core size). Both authors agree on the theoretical necessity of a $\dot{M} \propto M^{\alpha}$ dependence, with $\alpha=2.5$ for WNE and WC stars and $\alpha=0$ for WNL stars.

Maeder (1990a,b) calculates grids of evolutionary models for various values of the metallicity $Z$, notably those found in the Galaxy and the Magellanic Clouds, and scales $\dot{M} \propto Z^{0.5}$. He finds, depending on the value of $Z$, WR lifetimes of the order of $5 \times 10^{5} \mathrm{yr}$ and initial masses $M_{i}$ as low as $20 M_{\odot}$, to be compared with the observed minimum value of $25 M_{\odot}$ found by van der Hucht et al. (1988). WR/O, WC/WR and WC/WN number ratios following from Maeder's models compare remarkably well with the ratios observed in the Galaxy by van der Hucht et al. (1988), in the LMC (Azzopardi \& Breysacher 1985), and in M31 and M33 (Smith 1988). The comparison also indicates that in regions with lower $Z$ an increasing fraction of the observed WR stars has to originate from another 'channel' of WR formation, e.g., from binary evolution. The observed WN/WC ratio in the LMC requires $\dot{M}$-rates which are about $50 \%$ smaller than those in the solar neighborhood.

Maeder's model data confirm the overall general evolutionary sequence WNL $\rightarrow$ WNE $\rightarrow$ WCL $\rightarrow$ WCE $\rightarrow$ WO. Explicitly for binaries, studies by Moffat et al. (1990 and references therein) also point toward a continuous WCL $\rightarrow$ WCE/WO subtype evolution. Of the allowed subtype-evolution trails, based on the observed WR galactic distribution (van der Hucht et al. 1988), i.e.:

- $\quad$ at galactocentric radius $R<8.5 k p c$ : WNL $\rightarrow \mathrm{WCL}$ 
- $\quad$ at $R>6.5 k p c:$ WNL $\rightarrow$ WCE $\rightarrow$ WO

- $\quad$ and in general: WNE $\rightarrow$ no WC stars,

the first two trails agree very well with models with high $M_{i}$ and low $Z$ values, respectively.

The third trail corresponds only to lower $M_{i}$ at low $Z$, but may be somewhat relaxed since WNE stars and WCE stars do share some common galactocentric distances (Maeder $1990 \mathrm{~b}$ ). In spite of the different physics, the results of the models by Langer agree generally very well with those Maeder, both predicting similar surface abundances and, e.g., also the $\sim 3 \%$ fraction of WN/WC 'transition' stars classified by Conti \& Massey (1989).

Evolutionary models of massive close binaries including mass transfer and convective core overshooting, linking WR binaries with O-type binaries and X-ray binaries, have been calculated by, e.g., Doom \& De Greve (1983), De Greve \& Doom (1988), and De Greve et al. (1988), Although direct observational evidence of mass transfer is lacking, observed mass ratios and the non-evolved luminosity class of O-type components in some WR binaries point to some degree of rejuvenation by mass transfer. Even periodic mass transfer seems required, to explain the existence of the very eccentric $(e=0.84) \mathrm{WC} 7+04$ binary WR140 (De Greve, these proceedings). Duplicity constituting somehow an additional incentive for WR formation, be it by mass transfer or by tidal mixing, is also needed to explain the observed number ratios in regions with low $Z$ (Maeder 1990b). In a comparative study, Schulte-Ladbeck (1989) finds that present WR binary observations can better be reconciled with non-conservative models without convective core overshooting.

The indications for numerous WR stars in some irregular dwarf galaxies and blue compact emission line galaxies (see Table 2), as inferred from the intensity of their HeII 44686 emission, provide convincing evidence for massive starburst events or intermittent short bursts. The latter, coined 'lazy' galaxies by Kunth \& Joubert (1985), show WR stars in sites of higher metallicity and low effective temperature $(T<35 k K)$. Kunth \& Schild (1986) find further evidence for a correlation between the $\lambda 4686$ luminosity and the metallicity of the nebular gas, in addition to a correlation between the $\lambda 4686$ luminosity and the luminosity of the parent galaxy. Closer to Earth, a relationship between the WR star numbers and subclass distributions with metallicity in Local Group galaxies has been demonstrated by Smith (1988), making extragalactic WR research a powerful instrument for verification of the realism of evolutionary models.

\section{Concluding remarks}

All the above will be superseded this week by your own excellent reviews and contributions. It is with good confidence that I express the hope that this symposium will make proper use of the bonanza of observational and theoretical achievements of the past, and that we, on this inspiring island, together will come to new improvements and refinements of our understanding of those arresting physics laboratories, called Wolf-Rayet stars.

\section{References}

Abbott, D.C. 1982, Astrophys. J. 259, 282.

Abbott, D.C., Bieging, J.H., Churchwell, E., Torres, A.V. 1986, Astrophys. J. 303, 239.

Abbott, D.C., Conti, P.S. 1987, Ann. Rev. Astron. Astrophys. 25, 113. 
Acker, A., Stenholm B. 1990, Astron. Astrophys. Suppl. 86, 219.

Allen, D.A., Swings, J.P., Harvey, P.M. 1972, Astron. Astrophys. 20, 333.

Allen, D.A., Wright, A.E., Goss, W.M. 1976, Monthly Notices Roy. Astron Soc. $177,91$.

Armandroff, T.E., Massey, P. 1985, Astrophys. J. 291, 685.

Armandroff, T.E., Massey, P., Conti, P.S. 1986, in: C. de Loore, A.J. Willis, P. Laskarides (eds.): Luminous Stars and Associations in Galaxies, Proc. IAU Coll. No. 116 (Dordrecht: Reidel), p. 239.

Armus, L., Heckman, T.M., Miley, G.K. 1988 Astrophys. J. (Letters) 326, L45.

Azzopardi, M., Breysacher, J. 1979, Astron. Astrophys. 75, 120.

Azzopardi, M., Breysacher, J. 1985, Astron. Astrophys. 149, 213.

Azzopardi, M., Lequeux, J., Maeder, A. 1988, Astron. Astrophys. 189, 34.

Balona, L.A., Egan, J., Marang, F. 1989, Monthly Notices Roy. Astron. Soc. $240,103$.

Bandierra, R. Turolla, R. 1990, Astron. Astrophys. 231, 85.

Barlow, M.J., Smith, L.J., Willis, A.J. 1981, Monthly Notices Roy. Astron. Soc. 196, 101.

Barlow, M.J., Hummer, D.G. 1982, in: C. de Loore, A.J. Willis (eds.), Wolf-Rayet Stars: Observations, Physics, Evolution, Proc. IAU Symp. No. 99 (Dordrecht: Reidel), p. 387.

Blake, J.B., Dearborn, D.S.P. 1989, Astrophys. J. (Letters) 338, L17.

Bohannan, B., Conti, P.S., Massey,P. 1985, Astron. J. 90, 600.

Boksenberg, Willis, A.J., Searle, L. 1977, Monthly Notices Roy. Astron. Soc. 180, 15 P.

Breysacher, J. 1981, Astron. Astrophys. Suppl. 43, 209.

Caillault, J.-P., Chanan, G.A., Hefland, D.J., Patterson, J., Nousek, J.A., Takalo, L.O., Bathun, G.D., Becker, R.H. 1985, Nature 313, 376.

Campbell, A.W., Smith, L.J. 1986, in: C. de Loore, A.J. Willis, P. Laskarides (eds.): Luminous Stars and Associations in Galaxies, Proc. IAU Symp. No. 116 (Dordrecht: Reidel), p. 499.

Campbell, A.W., Terlevich, R., Melnick, J. 1986, Monthly Notices Roy. Astron. Soc. 223, 811,

Chiosi, C., Stalio, R. (eds.) 1981, Effects of Mass Loss on Stellar Evolution, Proc. IAU Coll. No. 59 (Dordrecht: Reidel).

Chiosi, C., Maeder, A. 1986, Ann. Rev. Astron. Astrophys. 24, 329.

Chu, Y.-H., Treffers, R.R., Kwitter, K.B. 1983, Astrophys. J. Suppl. 53, 937.

Chu, Y.-H. 1988, Publ. Astron. Soc. Pacific 100, 986.

Clayton, C.A. 1988, Monthly Notices Roy. Astron. Soc. 231, 191.

Cohen, M., Tielens, A.G.G.M., Bregman, J.D. 1989, Astrophys. J. (Letters) 344, L13.

Cohen, M., van der Hucht, K.A., Williams, P.M., Thé, P.S. 1991, Astrophys. J. (Letters), in press.

Conti, P.S., Massey, P. 1981, Astrophys. J. 249, 471.

Conti, P.S., Garmany, C.D., de Loore, C., Vanbeveren, D. 1983, Astrophys. J. 274, 302.

Conti, P.S. 1988, in: V.M. Blanco, M.M. Phillips (eds.), Progress and Opportunities in Southern Hemisphere Optical Astronomy, Proc. CTIO 25th Anniversary Symposium, A.S.P. Conf. Series Vol. 1, p. 100.

Conti, P.S, Underhill A.B. (eds.) 1988, O Stars and Wolf-Rayet Stars, NASA SP-497.

Conti, P.S., Massey, P. 1989, Astrophys. J. 337, 251.

Conti, P.S., Morris, P.W. 1990, Astron. J. 99, 898.

Conti, P.S., Vacca, W.D. 1990, Astron. J. 100, 431.

Conti, P.S., Massey, P., Vreux, J.-M. 1990, Astrophys. J. 354, 359.

Corso, G.J. 1975, Thesis, Northwestern University.

Cox, A.N., Cahn, J.H. 1988, Astrophys. J. 326, 804.

Davidson, K., Kinman, T.D. 1982, Publ. Astron. Soc. Pacific 94, 634.

Davidson, K., Moffat, A.F.J, Lamers, H. (eds.) 1989, Physics of Luminous Blue Variables, Proc. IAU Coll. No. 113 (Dordrecht: Kluwer).

De Greve, J.-P., Doom, C. 1988, Astron. Astrophys. 200, 79.

De Greve, J.-P., Hellings, P., van den Heuvel, E.P.J. 1988, Astron. Astrophys. 189, 74.

Deharveng, L., Caplan, J., Lequeux, J., Azzopardi, M., Breysacher, J., Tarenghi, M., Westerlund, B. 1988, Astron. Astrophys. Suppl. 73, 407.

Dinerstein, H.L., Shields, G.A. 1986, Astrophys. J. 311, 45.

D'Odorico, S., Baade, D., Kjär, K. (eds.) 1981, The Most Massive Stars, Proc. ESO Workshop (Garching: ESO).

D'Odorico, S., Rosa, M. 1981, Astrophys. J. 248, 1015.

D'Odorico, S., Rosa, M. 1982, Astron. Astrophys. 105, 410. 
D’Odorico, S., Benvenuti, P. 1983, Monthly Notices Roy. Astron. Soc. 203, 157.

D'Odorico, S., Rosa, M., Wampler, J. 1983, Astron. Astrophys. Suppl. 53, 97.

Doom, C., De Greve, J.-P. 1983, Astron. Astrophys. 120, 97.

Drissen, L., Lamontagne, R., Moffat, A.F.J., Bastien, P., Seguin, M. 1986, Astrophys. J. 304, 188.

Drissen, L., Moffat, A.F.J., Shara, M.M. 1989a, in: K. Davidson et al. (eds.), Physics of Luminous Blue Variables, Proc. IAU Coll. No. 113 (Dordrecht: Kluwer), p. 308.

Drissen, L., Robert, C., Lamontagne, R., Moffat, A.F.J., St-Louis, N., van Weeren, N., van Genderen, A.M. 1989b, Astrophys. J. 343, 426.

Dufour, R.J., Parker, R.A.R., Henize, K.G. 1988, Astrophys. J. 327, 859.

Dufour, R.G. 1989, in: S. Torres-Peimbert, J. Fierro (eds.), Star Forming Regions and Ionized Gas, Proc. 2nd Mexico-Texas Conf. on Astrophysics, Rev. Mexicana Astron. Astrof. $18,87$.

Durret, F., Bergeron, J., Boksenberg, A. 1985, Astron. Astrophys. 143, 347.

Durret, F., Tarrab, I. 1988, Astron. Astrophys. 205, 9.

Ensman, L.M., Woosley, S.E. 1988, Astrophys. J. 333, 754.

Firmani, C., Koenigsberger, G., Bisiacchi, G.F., Moffat, A. F.J., Isserstedt, J. 1980, Astrophys. J. 239, 607.

Freedman, W.L. 1985, Astron. J. 90, 2499.

Gamow, G. 1943, Astrophys. J. 98, 500.

Garmany, C.D., Massey, P., Conti, P.S. 1984, Astrophys. J. 278, 233.

Garmany, C.D (ed.) 1990, Intrinsic Properties of Hot Luminous Stars, Proc. Boulder-Munich Workshop, A.S.P. Conf. Series Vol. 7.

van Genderen, A.M., van der Hucht, K.A., Steemers, W.J.G. 1987, Astron. Astrophys. $185,131$.

van Genderen, A.M., van der Hucht, K.A., Larsen, I. 1990, Astron. Astrophys. $229,123$.

Gosset, E., Vreux, J.-M. 1990, Astron. Astrophys. 231, 100.

Gosset, E., Vreux, J.-M., Manfroid, J., Remy, M., Sterken, C. 1990, Astron. Astrophys. Suppl. 84, 377.

Hamann, W.-R., Schmutz, W. 1987, Astron. Astrophys. 174, 173.

Hamann, W.-R., Schmutz, W., Wessolowski, U. 1988, Astron. Astrophys. 194, 190.

Hamann, W.-R., Wessolowski, U., Schwarz, E., Dünnebeil, G., Schmutz, W. 1990, in: C.D. Garmany (ed.), Intrinsic Properties of Hot Luminous Stars, Proc. Boulder-Munich Workshop, A.S.P. Conf. Series Vol. 7, p. 259.

van den Heuvel, E.P.J. 1976, in: P. Eggleton, S. Mitton, J. Whelan (eds.), Structure and Evolution of Close Binary Systems, Proc. IAU Symp. No. 73 (Dordrecht: Reidel), p. 35.

Heydari-Malayeri, M, Melnick, J., Van Drom, E. 1990, Astron. Astrophys. (Letters) 236, L21.

Hidayat, B., Supelli, K., van der Hucht, K.A. 1982, in: C. de Loore, A.J. Willis (eds.), Wolf-Rayet Stars: Observations, Physics, Evolution, Proc. IAU Symp. No. 99 (Dordrecht: Reidel), p. 27.

Hidayat, B., Admiranto, A.G., van der Hucht, K.A. 1984, in: B. Hidayat, Z. Kopal, J. Rahe (eds.), Double Stars: Physical Properties and Generic Relations, Proc. IAU Coll. No. 80, Astrophys. Space Sci. 99, 175.

Hillier, D.J. 1987a, Astrophys. J. Suppl. 63, 947.

Hillier, D.J. 1987b, Astrophys. J. Suppl. 63, 965.

Hillier, D.J. 1988, Astrophys. J. 327, 822.

Hillier, D.J. 1989, Astrophys. J. 347, 392.

Hillier, D.J. 1990, in: C.D. Garmany (ed.), Intrinsic Properties of Hot Luminous Stars, Proc. BoulderMunich Workshop, A.S.P. Conf. Series Vol. 7, p. 340.

Hippelein, H., Fried, J.W. 1984, Astron. Astrophys. 141, 49.

van der Hucht, K.A., Conti, P.S., Lunström, I., Stenholm, B. 1981, Space Sci. Rev. 28, 22.

van der Hucht, K.A., Jurriens, T.A., Olnon, F.M., Thé, P.S., Wesselius, P.R., Williams, P.M. 1985, in: W. Boland, H. van Woerden (eds.), Birth and Evolution of Massive Stars and Stellar Groups, Proc. Symp. in honour of Adriaan Blaauw (Dordrecht: Reidel), p. 167.

van der Hucht, K.A., Cassinelli, J.P., Williams, P.M. 1986, Astron. Astrophys. 168, 111; $175,356$.

van der Hucht, K.A., Hidayat, B., Admiranto, A.G., Supelli, K.R., Doom, C. 1988, Astron. Astrophys. $199,217$.

van der Hucht, K.A. 1990, A Bibliography of Wolf-Rayet Literature 1980 - 1990, preprint.

van der Hucht, K.A., van Genderen, A.M., Bakker, P.R. 1990, Astron. Astrophys. 228, 108.

Hutchings, J.B., Massey, P., Bianchi, L. 1987, Astrophys J. (Letters) 322, L79. 
Hutsemekers, D., Surdej, J. 1984, Astron. Astrophys. 133, 209.

Jeffers, S., Weller, W.G. 1985, Astron. Astrophys. Suppl. 61, 173.

Johansson, L. 1987, Astron. Astrophys. 182, 179.

Johansson, L., Bergvall, N. 1988, Astron. Astrophys. 192, 81.

Keel, W.C. 1982, Publ. Astron. Soc. Pacific 94, 765.

Keel, W.C. 1987, Astron. Astrophys. 172, 43.

Khaliullin, Kh.F. 1974, Astron. Zh. 51, 395 (= Sov. Astron. 18, 229).

Khaliullin, Kh.F., Khaliullina, A.I., Cherepashchuk, A.M. 1984, Pis'ma Astron. Zh. 10, 600 (= Sov. Astron. Letters 10, 250).

Kudritzki, R.P., Hummer, D.G. 1990, Ann. Rev. Astron. Astrophys. 28, 303.

Kunth, D., Sargent, W.L.W. 1981, Astron. Astrophys. (Letters) 101, L5.

Kunth, D., Joubert, M. 1985, Astron. Astrophys. 142, 411.

Kunth, D., Schild, H. 1986, Astron. Astrophys. 169, 71.

Kwitter, K.B. 1981, Astrophys. J. 245, 154.

Kwitter, K.B. 1984, Astrophys. J. 287, 840.

Lamers, H., de Loore, C. (eds.) 1987, Instabilities in Luminous Early Type Stars, Proc. Workshop in honour of Cornelis de Jager (Dordrecht: Reidel).

Lamers, H., Maeder, A., Schmutz, W., Cassinelli, J.P. 1990, in: L.A. Willson, R. Stalio (eds.), Angular Momentum and Mass Loss for Hot Stars, NATO ASI Series Vol. C316 (Dordrecht: Kluwer), p. 349.

Langer, N. 1989a, Astron. Astrophys. 210, 93.

Langer, N. 1989b, Astron. Astrophys. 220, 135.

Langer, N. 1990, in: C.D. Garmany (ed.), Intrinsic Properties of Hot Luminous Stars, Proc. Boulder-Munich Workshop, A.S.P. Conf. Series Vol. 7, p. 328.

Leitherer, C., Chavarria, K. 1987, Astron. Astrophys. 175, 208.

Lequeux, J., Mancherat-Joubert, M., Deharveng, J.M., Kunth, D. 1981, Astron. Astrophys. $103,305$.

Lequeux, J., Meyssonnier, N., Azzopardi, M. 1987, Astron. Astrophys. Suppl. 67, 169.

de Loore, C., A.J. Willis (eds.) 1982, Wolf-Rayet Stars: Observations, Physics, Evolution, Proc. IAU Symp. No. 99 (Dordrecht: Reidel).

de Loore, C.W.H, Willis, A.J., Laskarides, P. (eds.) 1986, Luminous Stars and Associations in Galaxies, Proc. IAU Symp. No. 116 (Dordrecht: Reidel).

de Loore, C. 1988, Astron. Astrophys. 203, 71.

Lortet, M.C., Pitault, A. (eds.) 1983, Wolf-Rayet Stars: Progenitors of Supernovae? (Paris: l'Observatoire).

Lozinskaya, T.A. 1982, Astrophys. Space Sci. 87, 313.

Lundström, I., Stenholm, B. 1979, Astron. Astrophys. Suppl. 35, 303.

Lundström, I., Stenholm, B. 1984a, Astron. Astrophys. Suppl. 58, 163.

Lundström, I., Stenholm, B. 1984b, Astron. Astrophys. Suppl. 56, 43.

Lundström, I., Stenholm, B. 1984c, Astron. Astrophys. 138, 311.

Lundström, I., Stenholm, B. 1989, Astron. Astrophys. 218, 199.

Luo, D., McCray, R., Mac Low, M.-M. 1990 Astrophys. J. 362, 267.

Maeder, A., Lequeux, J., Azzopardi, M. 1980, Astron. Astrophys. (Letters) 90, L17.

Maeder, A. 1983, Astron. Astrophys. 120, 130.

Maeder, A. 1985, Astron. Astrophys. 147, 300.

Maeder, A., Meynet, G. 1987, Astron. Astrophys. 182, 243.

Maeder, A. 1990a, Astron. Astrophys. Suppl. 84, 139.

Maeder, A. 1990b, Astron. Astrophys., in press.

Massey, P. 1981, Astrophys. J. 246, 153.

Massey, P., Conti, P.S. 1983, Astrophys. J. 273, 576.

Massey, P. 1984, Astrophys. J. 281, 789.

Massey, P. 1985, Publ. Astron. Soc. Pacific 97, 5.

Massey, P., Armandroff, T.E., Conti, P.S. 1986, Astron. J. 92, 1303.

Massey, P., Conti, P.S., Armandroff, T.E. 1987a, Astron. J. 94, 1538.

Massey, P., Conti, P.S., Moffat, A.F.J., Shara, M.M. 1987b, Publ. Astron. Soc. Pacific 99, 816.

McCandliss, S.R. 1988, Thesis, Univ. of Colorado.

Meylan, G., Maeder, A. 1983, Astron. Astrophys., 124, 84. 
Moffat, A.F.J., Shara, M.M. 1983, Astrophys. J., 273, 544.

Moffat, A.F.J., Shara, M.M. 1987, Astrophys. J. 320, 266.

Moffat, A.F.J. 1988, in: G.V. Coyne, A.M. Magalhães, A.F.J. Moffat, R.E. Schulte-Ladbeck, S. Tapia, D.T., Wickramasinghe (eds.), Polarized Radiation of Circumstellar Origin (Vatican: Vatican Observatory), p. 607.

Moffat, A.F.J., Drissen, L., Lamontagne, R., Robert, C. 1988, Astrophys. J. 334, 1038.

Moffat, A.F.J., Niemela, V.S., Marraco, H.G. 1990, Astrophys. J. 348, 232.

Möllenhoff, C. 1981, Astron. Astrophys. 99, 341.

Moran,J.P., Davis, R.J., Bode, M.F., Taylor, A.R., Spencer, R.E., Argue, A.N., Irwin, M.J., Shanklin, J.D. 1989, Nature 340, 449.

Morgan, D.H., Good, A.R. 1990, Monthly Notices Roy. Astron. Soc. 243, 459.

Nugis, T., Pustyl'nik I. (eds.), 1988, Wolf-Rayet Stars and Related Objects, Proc. All-Union Conf., El'va, USSR, 1986, Tartu Astrofüüs. Obs. Teated No. 89.

Nussbaumer, H., Schmutz, W., Smith, L.J., Willis, A.J. 1982, Astron. Astrophys. Suppl. 47, 257.

Osterbrock, D.E., Cohen, R.D. 1982, Astrophys. J. 261, 64.

Poe, C.H., Friend, D.B., Cassinelli, J.P. 1989, Astrophys. J. 337, 888.

Pollock, A.M.T. 1987, Astrophys. J. 320, 283.

Prévot-Burnichon, M.L., Prévot, L., Rebeirot, E., Rousseau, J., Martin, N. 1981, Astron. Astrophys. 103, 83.

Prinja, R.K., Barlow, M.J., Howarth, I.D. 1990, Astrophys. J. 361, 607.

Rayo, J.F., Peimbert, M., Torres-Peimbert, S. 1982, Astrophys. J. 255, 1.

Robert, C., Moffat, A.F.J. 1990, in: C.D. Garmany (ed.), Intrinsic Properties of Hot Luminous Stars, Proc. Boulder-Munich Workshop, A.S.P. Conf. Series Vol. 7, p. 271,

Roberts, M.S. 1962, Astron. J. 67, 79.

Rosa, M., D’Odorico, S. 1982, in: C. de Loore, A.J. Willis (eds.), 'Wolf-Rayet Stars: Observations, Physics, Evolution', Proc. IAU Symp. No. 99 (Dordrecht: Reidel), p. 555.

Rosa, M., Solf, J. 1984, Astron. Astrophys. 130, 29.

Rosa, M., Joubert, M., Benvenuti, P. 1984, Astron. Astrophys. Suppl. 57, 361.

Rosa, M., D'Odorico, S. 1986, in: C.W.H. de Loore, A.J. Willis, P. Laskarides (eds.), Luminous Stars and Associations in Galaxies, Proc. IAU Symp. No. 116 (Dordrecht: Reidel), p. 355.

Rosa, M.R., Mathis, J.S. 1990, in: C.D. Garmany (ed.), Intrinsic Properties of Hot Luminous Stars, Proc. Boulder-Munich Workshop, A.S.P. Conf. Series Vol. 7, p. 135.

Rosa, M., Richter, O.-G. 1988, Astron. Astrophys. 192, 57.

Rosado, M. 1986, Astron. Astrophys. 160, 211.

Schmutz, W., Hamann, W.-R. 1986, Astron. Astrophys. (Letters) 166, L11.

Schmutz, W., Hamann, W.-R., Wessolowski, U. 1989, Astron. Astrophys. 210, 236.

Schmutz, W. 1990, in: C.D. Garmany (ed.), Intrinsic Properties of Hot Luminous Stars, Proc. BoulderMunich Workshop, A.S.P. Conf. Series Vol. 7, p. 117.

Schulte-Ladbeck, R.E. 1989, Astron. J. 97, 1471.

Schulte-Ladbeck, R.E., van der Hucht, K.A. 1989, Astrophys. J. 337, 872.

Shara, M.M., Moffat, A.F.J. 1986, in: C.W.H. de Loore, A.J. Willis, P. Laskarides (eds.), Luminous Stars and Associations in Galaxies, Proc. IAU Symp. No. 116 (Dordrecht: Reidel), p. 231.

Shore, S.N., Brown, D.N. 1988, Astrophys. J. 334, 1021.

Signore, M., Dupraz, C. 1990, Astron. Astrophys. (Letters) 234, L15 .

Sivertsen, S. 1981, Astron. Astrophys. Suppl. 43, 221.

Skillmann, E.D. 1985, Astrophys. J. 290, 449.

Smith, L.F. 1968a, Monthly Notices Roy. Astron. Soc. 140, 409.

Smith, L.F. 1968b, Monthly Notices Roy. Astron. Soc. 141, 317.

Smith, L.F., 1973, in: M.K.V. Bappu, J. Sahade (eds.), Wolf-Rayet and High-Temperature Stars, Proc. IAU Symp. No. 49 (Dordrecht: Reidel), p. 15.

Smith, L.F., Kuhi, L.V. 1981, An Atlas of Wolf-Rayet Line Profiles, JILA Report No. 117 (Boulder: Univ. of Colorado).

Smith, L.F. 1988, Astrophys. J. 327, 128.

Smith, L.F., Hummer, D.G. 1988, Monthly Notices Roy. Astron. Soc. 230, 511.

Smith, L.F., Maeder, A. 1989, Astron. Astrophys. 211, 71. 
Smith, L.F., Shara, M.M., Moffat, A.F.J. 1990, Astrophys. J. 358, 229.

Smith, L.F., Maeder, A. 1990, Astron. Astrophys., in press.

Smith, L.J., Willis, A.J. 1983, Astron. Astrophys. Suppl. 54, 229.

Smith, L.J., Pettini, M., Dyson, J.E., Hartquist, T.W. 1988, Monthly Notices Roy. Astron. Soc. $234,625$.

Smith, L.J. 1990, in: Proc. XIth Eur. Regional Astron. Meeting IAU (Cambridge: Univ. Press), in press.

St-Louis, N., Moffat, A.F.J., Drissen, L., Bastien, P., Robert, C. 1988, Astrophys. J. 330, 286.

St-Louis, N., Smith, L.J., Stevens, I.R., Willis, A.J., Garmany, C.D., Conti, P.S. 1989, Astron. Astrophys. 226, 249.

Taylor, M.-J., Chen, K.-Y. McNeill, J.D., Merrill, J.E., Oliver, J.P., Wood, F.B. 1988, Publ. Astron. Soc. Pacific 100, 1544.

Taylor, M.-J. 1990, Astron. J. 100, 1264.

Terlevich, R., Melnick, J. 1985, Monthly Notices Roy. Astron. Soc. 213, 841.

Thé, P.S. 1964, Contr. Bosscha Obs. No. 26.

Torres, A.V., Conti, P.S. 1984, Astrophys. J. 280, 181.

Torres, A.V., Conti, P.S., Massey, P. 1986, Astrophys. J. 300, 379.

Torres, A.V., Massey, P. 1987, Astrophys. J. Suppl. 65, 459.

Torres-Dodgen, A.V., Massey, P. 1988, Astron. J. 96, 1076.

Underhill, A.B. 1990, in: L.A. Willson, R. Stalio (eds.), Angular Momentum and Mass Loss for Hot Stars, NATO ASI Series Vol. C316 (Dordrecht: Kluwer), p. 353.

Underhill, A.B., Grieve, G.R., Louth, H. 1990,

Usov, V.V. 1990, Astroph. Space Sci. 167, 297. Publ. Astron. Soc. Pacific 102, 749.

Vacca, W.D., Torres-Dodgen, A.V. 1990, Astrophys. J. Suppl., in press.

Van Buren, D., McCray, R. 1988, Astrophys. J. (Letters) 329, L93.

Vreux, J.M., Dennefeld, M., Andrillat, Y. 1983, Astron. Astrophys. Suppl. 54, 437.

Vreux, J.-M. 1985, Publ. Astron. Soc. Pacific 97, 274.

Vreux, J.-M., Andrillat, Y., Gosset, E. 1985, Astron. Astrophys. 149, 337.

Vreux, J.-M. 1987, in: H. Lamers, C. de Loore (eds), Instabilities in Luminous Early Type Stars, Proc. Workshop in honour of Cornelis de Jager (Dordrecht: Reidel), p. 81.

Vreux, J.-M., Dennefeld, M., Andrillat, Y., Rochowicz, K. 1989, Astron. Astrophys. Suppl. $81,353$.

Vreux, J.-M., Andrillat, Y., Biemont, E. 1990, Astron. Astrophys. 238, 207.

Wampler, E.J. 1982, Astron. Astrophys. 114, 165.

Westerlund, B.E., Azzopardi, M., Breysacher, J., Lequeux, J. 1983, Astron. Astrophys. 123, 159.

Williams, P.M., Longmore, A.J., van der Hucht, K.A., Talevera, A., Wamsteker, W.M., Abbott, D.C., Telesco, C.M. 1985, Monthly Notices Roy. Astron. Soc. 215, 23P.

Williams, P.M., van der Hucht, K.A., Thé, P.S. 1987, Astron. Astrophys. 182, 91.

Williams, P.M., Eenens, P.R.J. 1989, Monthly Notices Roy. Astron. Soc. 240, 445.

Williams P.M., van der Hucht, K.A., Pollock, A.M.T., Florkowski, D.R., van der Woerd, H., Wamsteker, W.M. 1990a, Monthly Notices Roy. Astron. Soc. 243, 662.

Williams P.M., van der Hucht, K.A., Thé, P.S., Bouchet, P. 1990b, Monthly Notices Roy. Astron. Soc. 247, $18 \mathrm{P}$.

Willis, A.J. 1981, in: C. Chiosi, R. Stalio (eds.), Effects of Mass Loss on Stellar Evolution, Proc. IAU Coll. No. 59 (Dordrecht: Reidel), p. 27.

Willis, A.J., van der Hucht, K.A., Conti, P.S., Garmany, C.D. 1986, Astron. Astrophys. Suppl. $63,417$.

Willis, A.J., Garmany, C.D. 1987, in: Y. Kondo (ed.), Exploring the Universe with the IUE satellite (Dordrecht: Reidel), p. 157.

Willis, A.J., Howarth, I.D., Smith, L.J., Garmany, C.D., Conti, P.S. 1989, Astron. Astrophys. Suppl. 77, 269.

Willson, L.A., Stalio, R. (eds) 1990, Angular Momentum and Mass Loss for Hot Stars, NATO ASI Series Vol. C\$16 (Dordrecht: Kluwer).

Wolf, C.J.E., Rayet, G. 1867, Comptes Rendus 65, 292.

Wray, J.D., Corso, G.J. 1972, Astrophys. J. 172, 577.

Wright, A.E., Barlow, M.J. 1975, Monthly Notices Roy. Astron. Soc. 170, 41.

Zinnecker, H. 1987, in: T.X. Thuan, T. Montmerle, J. Tran Thanh Van (eds.), Starbursts and Galactic Evolution, Proc XXIInd Rencontres de Moriond (Gif-sur-Yvettes: Editions Frontières), p. 165. 